\title{
Roles of mitochondrial ROS and NLRP3 inflammasome in multiple ozone-induced lung inflammation and emphysema
}

Feng $\mathrm{Li}^{1,2+}$, Mengmeng $\mathrm{Xu}^{1 \dagger}$, Muyun Wang ${ }^{1 \dagger}$, Lei Wang ${ }^{2}$, Hanying Wang ${ }^{2}$, Hai Zhang ${ }^{1}$, Yuqing Chen ${ }^{1}$, Jicheng Gong ${ }^{3,4}$, Junfeng(Jim) Zhang ${ }^{3,5}$, lan M. Adcock ${ }^{6,7}$, Kian Fan Chung ${ }^{6}$ and Xin Zhou ${ }^{2 *}$

\begin{abstract}
Background: Mitochondrial damage leading to oxidant stress may play an important role in the pathogenesis of airflow obstruction and emphysema. NLPR3 inflammasome can be activated by mitochondrial ROS (mtROS) and other stimuli. We examined the importance of mtROS and NLRP3 inflammasome and their interactions in multiple ozone-induced lung inflammation and emphysema.

Methods: C57/BL6 mice were exposed to ozone ( $2.5 \mathrm{ppm}, 3 \mathrm{~h}$ ) or filtered air twice a week over 6 weeks. MitoTEMPO (20 mg/kg), an inhibitor of mtROS, and VX765 (100 mg/kg), an inhibitor of caspase-1 activity, were administered by intraperitoneal or intragastric injection respectively $1 \mathrm{~h}$ prior to each ozone exposure for 6 weeks.

Results: Ozone-exposed mice had increased bronchoalveolar lavage (BAL) total cells and levels of IL-1 $\beta, \mathrm{KC}$ and IL-6, augmented lung tissue inflammation scores, enhanced oxidative stress with higher serum 8-OHdG concentrations, emphysema with greater mean linear intercept $(L m)$, airway remodeling with increased airway smooth muscle mass and airflow limitation as indicated by a reduction in the ratio of forced expiratory volume at 25 and 50 milliseconds to forced vital capacity ( $\mathrm{FEV}_{25} / \mathrm{FVC}, \mathrm{FEV}_{50} / \mathrm{FVC}$ ). Both MitoTEMPO and VX765 reduced lung inflammation scores, cytokine levels, oxidative stress and increased mitochondrial fission proteins. VX765 also attenuated emphysema, airway remodeling and airflow limitation. MitoTEMPO inhibited the increased expression of mitochondrial complex II and IV and of NLPR3 while VX765 inhibited the expression and activity of NLRP3 and caspase-1 pathway in the lung.
\end{abstract}

Conclusions: Both mtROS and NLRP3 inflammasome play a role in ozone-induced lung inflammation while only NLRP3 is involved in ozone-induced emphysema.

Keywords: Mitochondrial ROS, NLRP3 inflammasome, Ozone, Lung inflammation, Emphysema

\section{Background}

Chronic obstructive pulmonary disease (COPD) is a major lung disease currently affecting 384 million people worldwide, with a global prevalence of $11.7 \%$ [1]. The pathogenetic features of COPD, caused by cigarette smoke and other noxious particles or gases, include obstructive bronchiolitis and emphysema, which both lead to airflow limitation and respiratory symptoms [2]. The

* Correspondence: xzhou53@163.com

${ }^{\dagger}$ Feng Li, Mengmeng Xu and Muyun Wang contributed equally to this work. ${ }^{2}$ Department of Respiratory Medicine, Shanghai First People's Hospital,

Shanghai Jiaotong University, No.100, Haining Road, Shanghai 200080, China Full list of author information is available at the end of the article underlying mechanisms of COPD are not fully elucidated. However, oxidant-antioxidant imbalance or oxidative stress, due to over-production of reactive oxygen species (ROS) in excess of the antioxidant defenses, is a predominant mechanism for COPD [3].

Mitochondria are double membrane bound organelles that exist in most eukaryotic organisms. The morphology of mitochondria is regulated by fission and fusion. The former is mediated by dynamin related protein 1 (DRP1), Fission 1 (FIS1) and mitochondrial fission factor (MFF), while the latter is controlled by mitofusins 1 and 2 (MFN1 and MFN2), and optic atrophy protein 1 (OPA1) [4]. Mitochondria play an important role in production of adenosine triphosphate (ATP) and mitochondrial ROS (mtROS) [5]. Dysfunctional

(c) The Author(s). 2018 Open Access This article is distributed under the terms of the Creative Commons Attribution 4.0 International License (http://creativecommons.org/licenses/by/4.0/), which permits unrestricted use, distribution, and 
mitochondria influences airway contractility, gene expression, oxidative stress, immune response, cell proliferation, apoptosis and metabolism that are all implicated in COPD. Therefore, mitochondrial dysfunction is increasingly recognized as being involved in the pathogensis of COPD [6-8].

The nucleotide binding domain leucine-rich repeat-containing receptor (NLR) family members are cytosolic receptors that can sense pathogen- and damage -associated signaling, of which NLRP3 is the most widely characterized member. NLRP3 is proposed to play a role in the development of COPD [9]. Activation of the NLRP3 inflammasome involves a priming signal to upregulate the expression of NLRP3, and then the assembly of a multi-protein complex consisting of NLRP3 and pro-caspase-1, which enables caspase- 1 activation and IL-1 $\beta$ maturation [10]. Mitochondrial dysfunction can also trigger NLRP3 activation, as supported by the co-localization of activated NLRP3 with mitochondria [11] and the finding that oxidized mitochondrial DNA is a direct ligand of NLRP3 [12]. In addition, other triggers such as lipopolysaccharide and danger signals such as ATP, hyaluronan and necrotic cells activate the NLPR3 inflammasome [13-15].

Ozone $\left(\mathrm{O}_{3}\right)$ is an environmental pollutant that has been associated with worsening symptoms, increased hospitalizations and emergency visits in patients with COPD [16-18].We previously showed that repeated ozone exposure in mice led to chronic inflammation, emphysema and airflow limitation, all hallmark features of COPD [19, 20]. However, the molecular mechanisms of these effects are still not well established and an understanding of these may be relevant to the mechanisms underlying COPD. We hypothesize that NLRP3 inflammasome activation particularly by mtROS may be an important pathway in the development of COPD and therefore, inhibition of mtROS or of NLRP3 pathways may prevent ozone-induced features of COPD. We studied whether an mtROS inhibitor or a caspase-1 inhibitor could modulate the lung inflammation, emphysema, and airflow obstruction seen in the multiple ozone exposure mouse model.

\section{Methods}

\section{Ozone exposure and inhibitor administration}

The experimental procedures were approved by the Laboratory Animal Ethics Committee of Shanghai Chest Hospital. Eight-ten weeks male C57/BL6 mice (Shanghai Super- B\&K Laboratory Animal Corp. Ltd., Shanghai, China) were housed in specific-pathogen-free (SPF) conditions with food and water supplied ad libitum. Mice were exposed to ozone $(2.5 \mathrm{ppm})$ or filtered air for $3 \mathrm{~h}$, twice a week over 6 weeks as previously described $[19,20]$. Phosphate buffered saline (PBS, as vehicle) and MitoTEMPO (mtROS inhibitor, $20 \mathrm{mg} / \mathrm{kg}$, Sigma-Aldrich, St. Louis, MO, USA) dissolved in PBS and administered intraperitoneally. VX765 (caspase-1 inhibitor, $100 \mathrm{mg} / \mathrm{kg}$, Selleck, Houston, TX, USA) was dissolved in distilled water containing $0.5 \%$ sodium salt of carboxy methylellucose and $0.1 \%$ Tween- 80 and administered intragastrically to mice one hour before each exposure twice a week for 6 weeks. The detailed experimental protocol was outlined in Fig. 1.

To examine the effect of MitoTEMPO or VX765 on control mice, additional experiments were performed in which air-exposed mice were treated with 3 weeks of MitoTEMPO or VX765. An additional file shows this in more detail [see Additional file 1].

\section{Lung function}

After anesthesia with injection of ketamine $(100 \mathrm{mg} / \mathrm{kg})$ and xylazine $(10 \mathrm{mg} / \mathrm{kg})$, mice were tracheostomized and placed in a whole-body plethysmograph (EMMS, Hants, UK) with a ventilator to measure inspiratory capacity (IC), functional residual capacity (FRC), total lung capacity (TLC), forced vital capacity (FVC), forced expiratory volume in first 25 and 50 milliseconds of exhalation $\left(\mathrm{FEV}_{25}, \mathrm{FEV}_{50}\right)$ and chord compliance (Cchord) as previously described $[19,20]$.

\section{Measurement of bronchoalveolar lavage fluid and blood}

Following terminal anaesthesia, bronchoalveolar lavage (BAL) fluid and blood were collected as previously

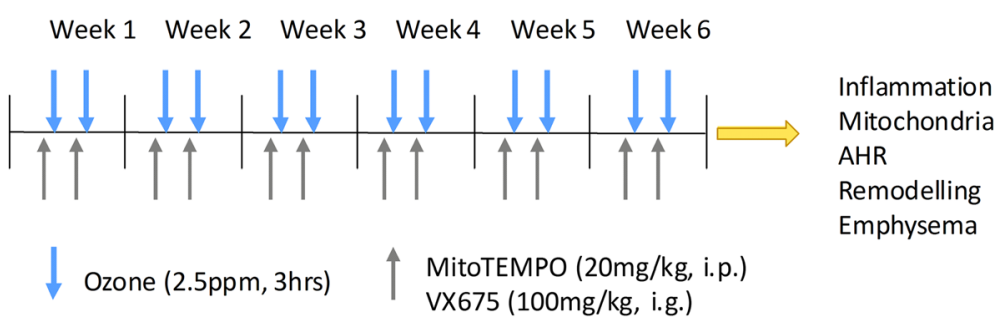

Fig. 1 Schematic diagram of the experimental protocol. Mice were exposed to ozone $(2.5 \mathrm{ppm})$ or filtered air for $3 \mathrm{~h}$, twice a week over 6 weeks. Mice were intraperitoneally injected with PBS or MitoTEMPO ( $\mathrm{mtROS}$ inhibitor, $20 \mathrm{mg} / \mathrm{kg}$, dissolved in PBS) or orally fed by gavage with VX765 (caspase-1 inhibitor, $100 \mathrm{mg} / \mathrm{kg}$, dissolved in distilled water containing $0.5 \%$ sodium salt of carboxy methylellucose and $0.1 \%$ Tween- 80 ) 1 hour before each exposure, twice a week for 6 weeks 
described [19, 20]. Total cell counts were determined using a hemocytometer, and differential cell counts from cytospin preparations were measured under a microscope. BAL malondialdehyde (MDA) was measured using HPLC-fluorescence technique and serum 8-hydroxy-2'deoxyguanosine (8-OHdG) was analyzed using HPLC-MSMS technique as previously described [19, 20]. Levels of chemokine (C-X-C motif) ligand 1 (CXCL1, KC), interleukin-6 (IL-6) and interleukin-1 $\beta$ (IL-1 $\beta$ ) in the BAL fluid were measured using enzyme-linked immunosorbent assay kits (R\&D systems, China Co. Ltd., Shanghai, China) according to manufacturers' instructions.

\section{Histological analysis}

The left lung was inflated with $4 \%$ paraformaldehyde under $25 \mathrm{~cm}$ of water pressure and then embedded in paraffin. $5 \mu \mathrm{m}$ sections were cut and stained with haematoxylin and eosin (H\&E). The mean linear intercept (Lm), a measure of interalveolar septal wall distance, was determined using a reticule with 5 lines (each $500 \mu \mathrm{m}$ long), with 10 fields per section assessed at random. Fields with airways or vessels were avoided. Lm was calculated by dividing the length of the line by the number of alveolar wall and grid line interceptions counted as described previously $[19,20]$.

Lung inflammatory response was scored on a $0-3$ scale as previously described [20]: 0 , no inflammation was detectable; 1 , occasional cuffing with inflammatory cells; 2, most bronchi or vessels were surrounded by a thin layer (one to five cells thick) of inflammatory cells; 3 , most bronchi or vessels were surrounded by a thick layer (more than five cells thick) of inflammatory cells. Lung inflammation was defined as the average of the peribronchial and perivascular inflammation scores.

To assess the change in the components of airway structure, the area of airway epithelium (Wae), airway smooth muscle (Wam), total airway wall (Wat), and the perimeter of basement membrane $(\mathrm{Pbm})$ were measured in H\&E-stained sections using a Motic digital microscope image analysis system (Motic China Group, Xiamen, Fujian, China) and parameters were calculated as $\mathrm{Wae} / \mathrm{Pbm}, \mathrm{Wam} / \mathrm{Pbm}$ and $\mathrm{Wat} / \mathrm{Pbm}$ as previously described $[19,20]$.

\section{Lung mtROS analysis and caspase- 1 activity}

Mitochondria were extracted from fresh lung tissues using a Mitochondria Isolation Kit for Tissue (Beyotime, Haimen, Jiangsu, China) with protease inhibitors using a Dounce tissue grinder following the manufacturer's instructions as described before [21], and then quantified by BCA analysis (Thermo Fisher Scientific). Equal amounts of mitochondrial extract were incubated with $5 \mu \mathrm{M}$ MitoSOX ${ }^{\mathrm{mm}}$ Red (Invitrogen) for $10 \mathrm{~min}$ at $37^{\circ} \mathrm{C}$ and protected from the light. Red fluorescence was measured at $510 / 580 \mathrm{~nm}$ using a Flexstation ${ }^{\circ} 2$ fluorescence reader (Molecular Devices, San Jose, CA, USA). The levels of caspase- 1 activity in mouse lung tissue were detected using a commercial assay kit (Beyotime) and measured at $405 \mathrm{~nm}$ by a microplate reader following the manufacturer's instructions as described before [22].

\section{Western blot}

Equal amounts of mitochondrial extract or lung homogenate were separated by $10 \%$ SDS-PAGE (Beyotime) and electrophoretically transferred to nitrocellulose membranes. The membranes were blocked, then incubated with primary antibodies against total OXPHOS antibody cocktail (Abcam, Cambridge, MA, USA), voltage-dependent anion channel (VDAC, Abcam), DRP1 (Cell Signaling Technologies - CST, Beverly, MA, USA), MFF (CST), MFN2 (CST), OPA1 (CST), GAPDH (CST), NLRP3 (CST), caspase-1 (Abcam) and tubulin (CST) for blot detection. VDAC, GAPDH and tubulin were used as respective loading controls. The density was quantitated using a densitometer.

\section{Statistical analysis}

All results were expressed as mean \pm S.E.M. One-way analysis of variance (ANOVA) with Bonferroni post-test or Dunnett T3 post-test analysis performed for comparisons between multiple groups using SPSS 20.0 software. $P<0.05$ was considered statistically significant.

\section{Results \\ Lung function}

Lung volume parameters (IC, FRC and TLC) and compliance (Cchord) were increased and airflow volume ratios $\left(\mathrm{FEV}_{25} / \mathrm{FVC}, \quad \mathrm{FEV}_{50} / \mathrm{FVC}\right)$ were decreased in ozone-exposed mice compared with control mice. Treatment with VX765 inhibited the increase in IC, FRC, TLC and Cchord, and prevented the decrease in $\mathrm{FEV}_{25} / \mathrm{FVC}$ and $\mathrm{FEV}_{50} / \mathrm{FVC}$, while treatment with MitoTEMPO showed no significant effect on any of these parameters (Fig. 2a-f).

\section{BAL fluid cells and cytokines and lung inflammatory socres}

Total BAL cell counts, including macrophages, lymphocytes, neutrophils and eosinophils, were increased in ozone-exposed mice compared with control mice. Treatment with VX765 decreased the numbers of total cells, macrophages, neutrophils and eosinophils. In contrast, treatment with MitoTEMPO did not affect total or differential cell counts although there was a trend towards reduced counts except for lymphocytes (Fig. 3a).

Increased lung inflammation scores with inflammatory cell infiltrates around bronchi or vessels in lung sections were observed in ozone-exposed mice compared with 

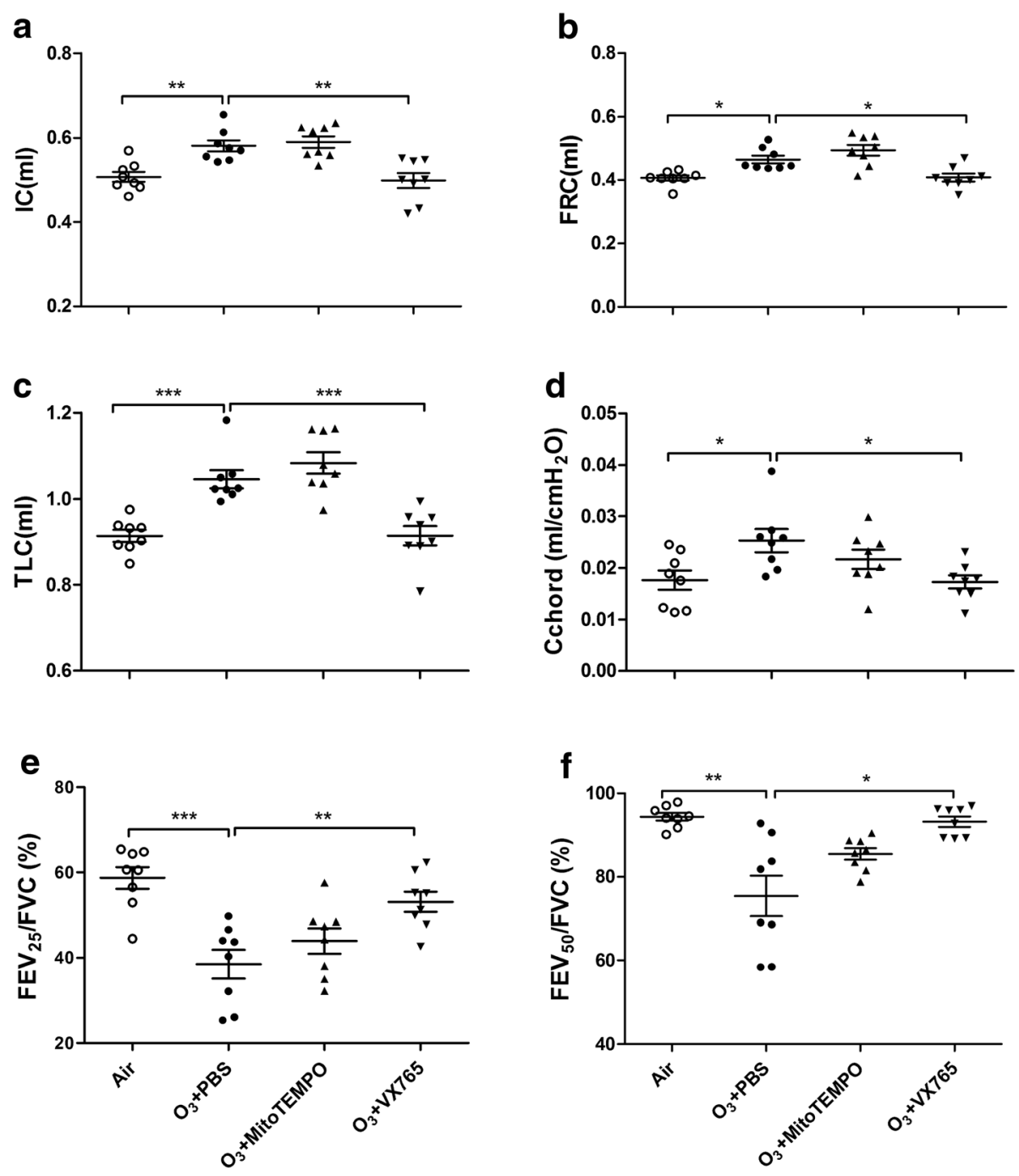

Fig. 2 Effects of MitoTEMPO and VX765 on multiple ozone exposure-induced lung function changes. The top panel indicates the experimental plan. Drugs were delivered $1 \mathrm{~h}$ prior to ozone exposure. Lung function analysis was composed of inspiratory capacity (IC) (a), functional residual capacity (FRC) (b), total lung capacity (TLC) (c), chord compliance (Cchord) (d), and percentage of forced expiratory volume (FEV) in first 25 and 50 ms of fast expiration ( $F E V_{25}$ and $\mathrm{FEV}_{50}$ ) of forced vital capacity $(\mathrm{FVC})(\mathbf{e}, \mathbf{f}) .{ }^{*} P<0.05$, ${ }^{* *} P<0.01,{ }^{* * *} P<0.001$. One-way analysis of variance (ANOVA) with a Bonferroni or Dunnett's T3 post-test analysis was performed for comparisons between multiple groups

control mice. Treatment with MitoTEMPO and VX765 reduced lung inflammation scores (Fig. 3b).

Increases in the level of IL-1 $\beta, \mathrm{KC}$ and IL-6 were observed in ozone-exposed mice compared to control mice. MitoTEMPO treatment caused a reduction in the levels of KC and IL- 6 and had a non-significant reduction in IL-1 $\beta$ concentration. VX765 treatment significantly decreased the levels of KC, IL-6 and IL-1 $\beta$ (Fig. 3c-e).

\section{Oxidative stress and lung caspase- 1 activity}

There was no increase in BAL MDA in ozone-exposed mice compared to control mice. Neither MitoTEMPO nor VX765 affected MDA concentrations (Fig. 4a). In contrast, levels of serum 8-OHdG were increased in ozone-exposed mice compared to control mice. MitoTEMPO and VX765 intervention both reduced serum 8-OHdG levels (Fig. 4b). Serum 8-OHdG was negatively correlated with $\mathrm{FEV}_{25} / \mathrm{FVC}$ $(r=-0.4021, P<0.05), \mathrm{FEV}_{50} / \mathrm{FVC}(r=-0.3815, \mathrm{P}<0.05)$, and positively correlated with BAL total cells $(r=0.4283$, $P<0.05)$, macrophages $(r=0.4221, \mathrm{P}<0.05)$, neutrophils $(r=0.4462, \mathrm{P}<0.05)$, eosinophils $(r=0.4494, P<0.01)$, IL-1 $\beta(r=0.5382, \mathrm{P}<0.01), \mathrm{KC}(r=0.5743, P<0.001)$ and IL-6 $(r=0.4147, P<0.05)$.

Increased mtROS in lung was found in ozone-exposed mice compared to control mice. Treatment with MitoTEMPO and VX765 reduced mtROS (Fig. 4c). Lung mtROS levels were positively correlated with 8-OHdG $(r=0.4672, P<0.01)$ and negatively correlated 

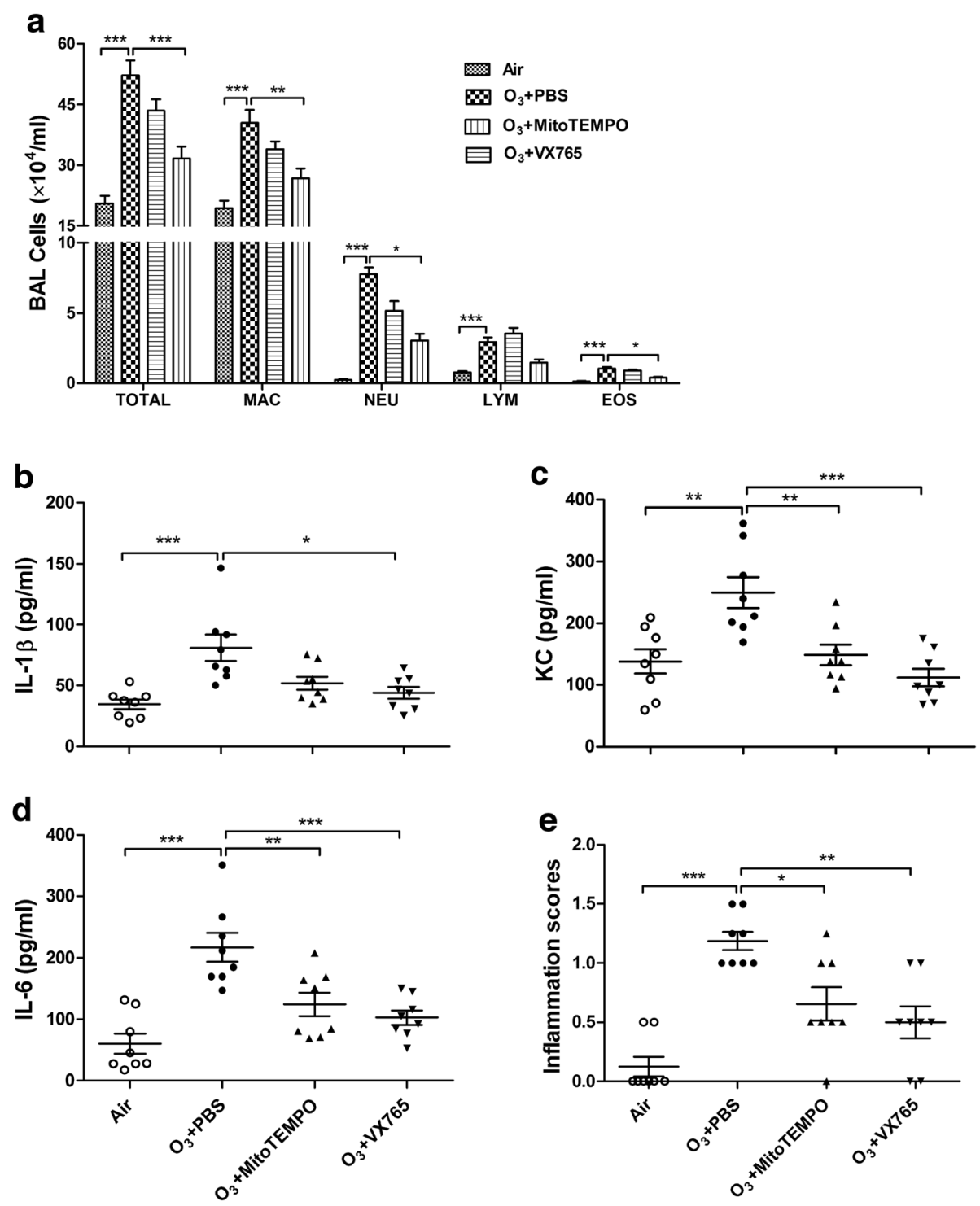

Fig. 3 Effects of MitoTEMPO and VX765 on multiple ozone exposure-induced airway inflammation and and lung inflammation. a Effects of MitoTEMPO and VX765 on multiple ozone exposure-induced increased cell numbers in bronchoalveolar lavage (BAL) fluid, including total cells (TOTAL), macrophages (MAC), lymphocytes (LYM), neutrophils (NEU) and eosinophils (EOS). Each bar represents the mean \pm SEM. b-d Effects of MitoTEMPO and VX765 on multiple ozone exposure-induced increased levels of cytokines in BAL fluid, including IL-1 $\beta$ (b), KC (c) and IL-6 (d). e Effects of MitoTEMPO and VX765 on multiple ozone exposure-induced inflammation scores in lung tissue. ${ }^{*} P<0.05,{ }^{*} P<0.01,{ }^{* * *} P<0.001$.

One-way analysis of variance (ANOVA) with Bonferroni or Dunnett's T3 post-test analysis was performed for comparisons between multiple groups

with FEV25/FVC $(r=-0.4469, P<0.05)$, FEV50/FVC $(r=-0.5537, \mathrm{P}<0.01)$. Lung mtROS also positively correlated with BAL total cells $(r=0.4534, \mathrm{P}<0.01)$, macrophages $(r=0.4178, \mathrm{P}<0.05)$, neutrophils $(r=0.5622$, $P<0.001)$, eosinophils $(r=0.4229, \quad \mathrm{P}<0.05)$, IL-1 $\beta$ $(r=0.4499, \mathrm{P}<0.01), \mathrm{KC}(r=0.5996, \mathrm{P}<0.001)$ and IL-6 $(r=0.4347, \mathrm{P}<0.05)$,

There was no difference in caspase- 1 activity in ozone-exposed mice compared to control mice. Treatment with VX765 reduced caspase-1 activity, while treatment with MitoTEMPO showed no effect (Fig. 4d).

\section{Histological analysis}

Lung alveolar enlargement after ozone exposure is depicted in Fig. 5a-d. There were increases in Lm, Wam and Wat in ozone-exposed mice compared with control mice. Treatment with VX765 prevented the increases of Lm, ASM and Wat in ozone-exposed mice. However, MitoTEMPO 

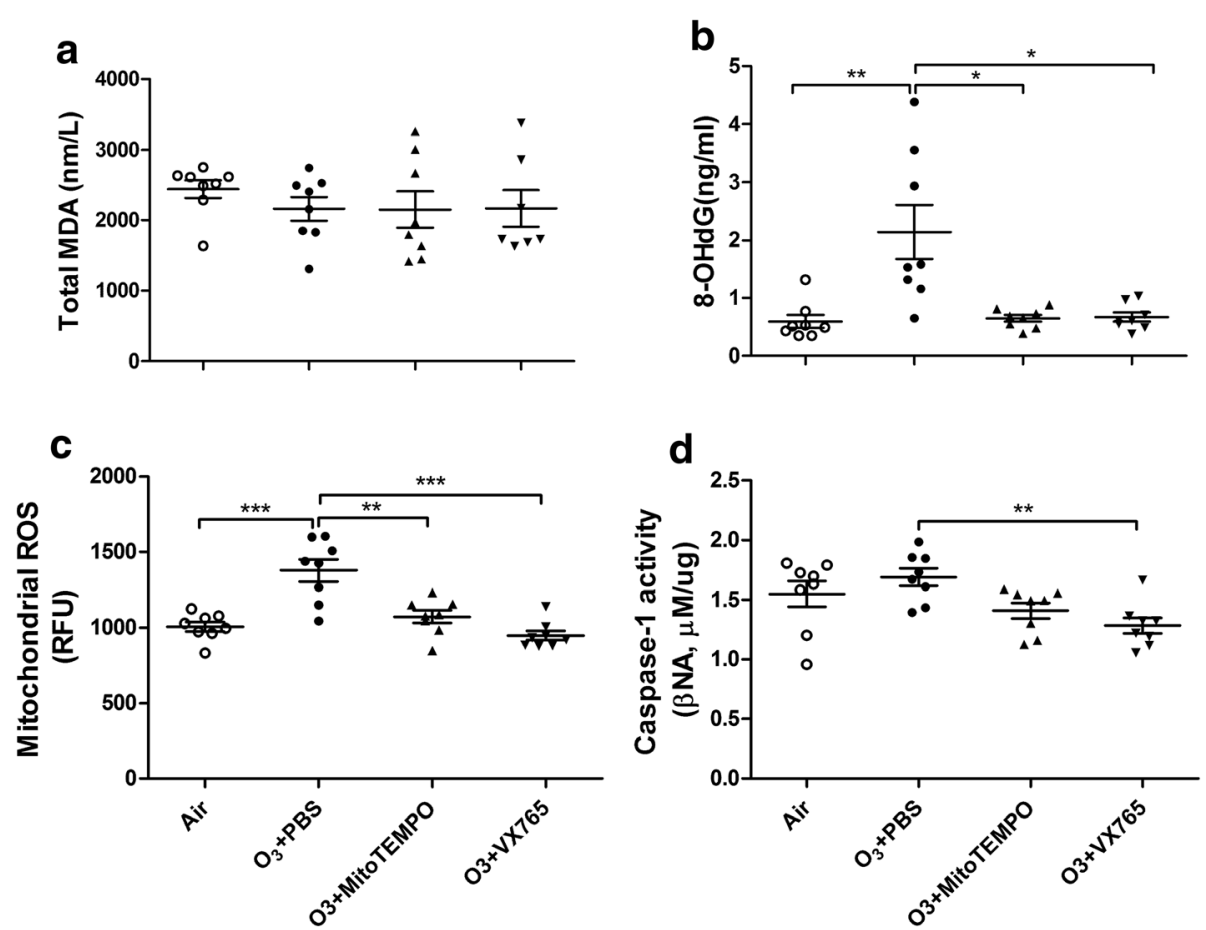

Fig. 4 Effects of MitoTEMPO and VX765 on multiple ozone exposure-induced increases in malonaldehyde in BAL fluid (a), 8-hydroxy-deoxyguanosine (8-OHdG) in serum (b), mitochodrial ROS (c) and caspase-1 activity (d) in lung tissue. ${ }^{*} P<0.05$, ${ }^{* *} P<0.01,{ }^{* *} P<0.001$. One-way analysis of variance (ANOVA) with Bonferroni or Dunnett's T3 post-test analysis was performed for comparisons between multiple groups

treatment affected none of these histological parameters (Fig. 5e, g, h). Lm was positively correlated with 8-OHdG $(r=0.5407, P<0.01)$ and mtROS $(r=0.4288$, $P<0.01)$. In addition, Wam $/ \mathrm{Pbm}(r=0.5859, P<0.001)$ and Wat $/ \mathrm{Pbm}(r=0.4610, P<0.01)$ were positively correlated with mtROS.

\section{Expression of mitochondrial OXPHOS, mitochondria- related proteins, NLRP3 \& caspase-1}

There was increased expression of mitochondrial OXPHOS complexes II and IV in ozone-exposed mice compared with control mice. MitoTEMPO treatment inhibited the expression of mitochondrial complex II and IV in the lung tissue whilst VX765 treatment showed no effect (Fig. 6a, d, e). Mitochondrial complex II expression was positively correlated with $\mathrm{mtROS}(r=0.4466, P<0.05)$, and mitochondrial complex IV expression was positively correlated with 8-OHdG $(r=0.4062, P<0.05)$.

The expression of DRP1 and MFF was increased in ozone-exposed mice compared to control mice and both MitoTEMPO and VX765 treatment inhibited the expression of DRP1 and MFF (Fig. 7a, b). The expression of MFN2 and OPA1 was unchanged in ozone-exposed mice compared to control mice and neither MitoTEMPO nor VX765 affected MFN2 or OPA1 expression (Fig. 7c, d).
DRP1 expression was positively correlated with 8-OHdG $(r=0.4938, P<0.01)$ and $\mathrm{mtROS}(r=0.3498, \mathrm{P}<0.05)$ whilst MFF expression was positively correlated with 8-OHdG $(r=0.5319, P<0.01)$ and mtROS $(r=0.4319$, $P<0.05)$.

The expression of NLPR3 and caspase-1 was increased in ozone-exposed mice compared to control mice. MitoTEMPO intervention inhibited the expression of NLRP3, while VX765 treatment inhibited the expression of NLPR3 and caspase-1 (Fig. 7e, f). NLRP3 expression was positively correlated with 8-OHdG $(r=0.4081, P<0.01)$ and $m$ tROS $(r=0.5772, P<0.001)$, and caspase- 1 expression was positively correlated with 8-OHdG $(r=0.6376, \mathrm{P}<0.001)$ and $\operatorname{mtROS}(r=0.4929, P<0.01)$.

The overall effects of MitoTEMPO and VX-765 on ozone-induced inflammation, lung function and emphysema are summarized in Table 1.

\section{Discussion}

In the present study, both MitoTEMPO and VX765 reduced multiple ozone-induced features including lung inflammation, oxidative stress, and increased mitochondrial fission proteins. In addition, VX765 reduced emphysema, airway remodeling and airflow limitation that were not affected by MitoTEMPO. MitoTEMPO specifically 

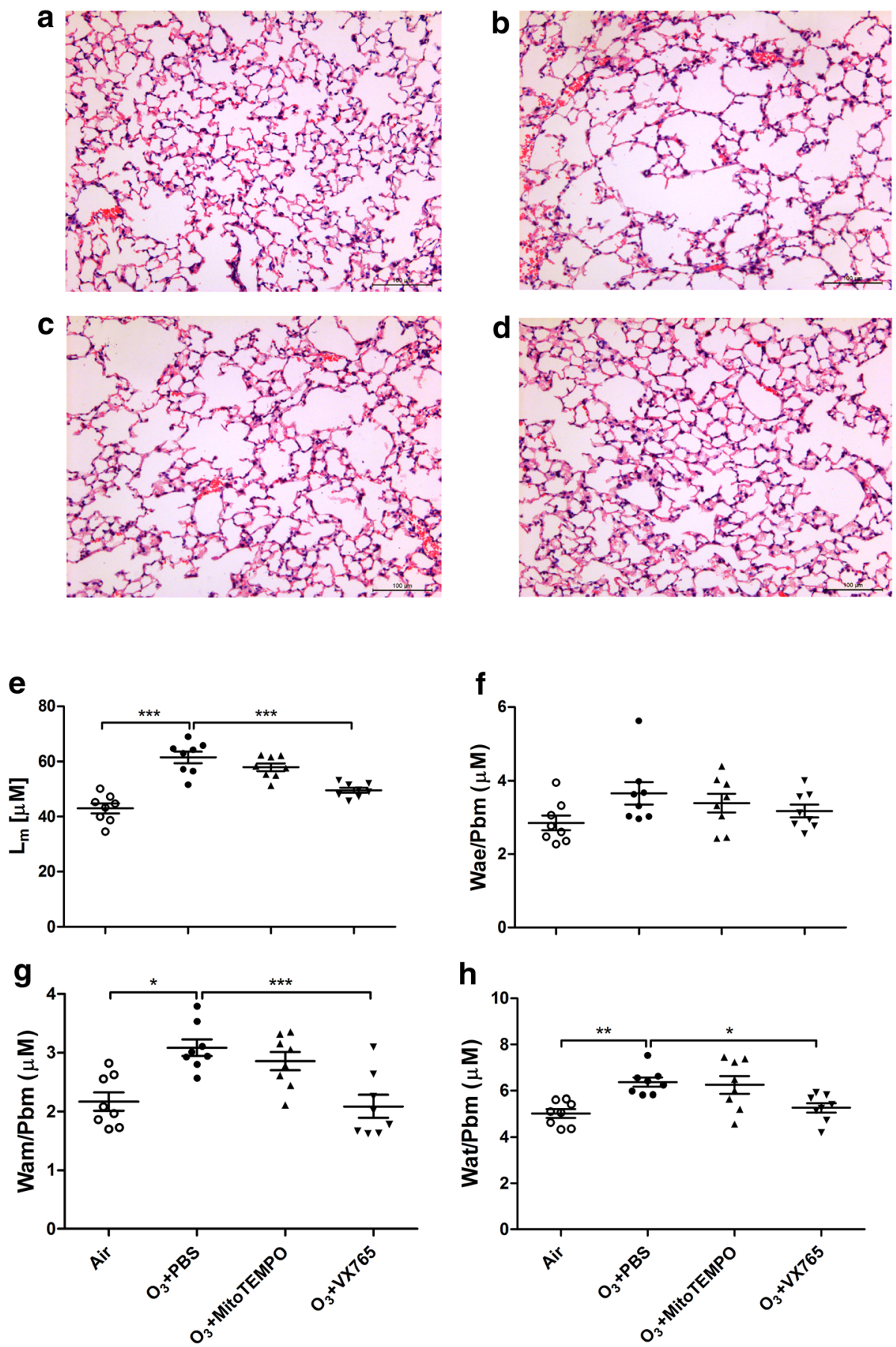

Fig. 5 Representative photomicrographs of lung alveolar spacesin haematoxylin-eosin-stained sections of air-exposed mice (a),ozone-exposed mice (b), MitoTEMPO-treated ozone-exposed mice (c), VX765-treated ozone exposed mice(d). (bar = $100 \mu \mathrm{m})$. Effects of MitoTEMPO and VX765 on multiple ozone exposure-induced increase in mean linear intercept $\left(\mathrm{L}_{\mathrm{m}}\right)(\mathbf{e})$ and changes in airway structure including thickness of airway epithelial layer $(\mathrm{Wae} / \mathrm{Pbm})(\mathbf{f})$, airway smooth muscle $(\mathrm{Wam} / \mathrm{Pbm})(\mathbf{g})$ and total airway wall $(\mathbf{h}) .{ }^{*} P<0.05,{ }^{* *} P<0.01$, ${ }^{* * *} P<0.001$. One-way analysis of variance (ANOVA) with Bonferroni or Dunnett's T3 post-test analysis was performed for comparisons between multiple groups

inhibited the expression of mitochondrial complexes II and IV and of NLRP3, while VX765 inhibited the expression and activity of NLRP3-caspase-1 pathway in the lung tissue. These data suggest that both mitochondrial dysfunction and NLRP3 activation may be involved in the pathogenesis of COPD. Targeting mtROS ameliorated some, but not all, of the features modulated by inhibition of the downstream target NLRP3 inflammasome/caspase 


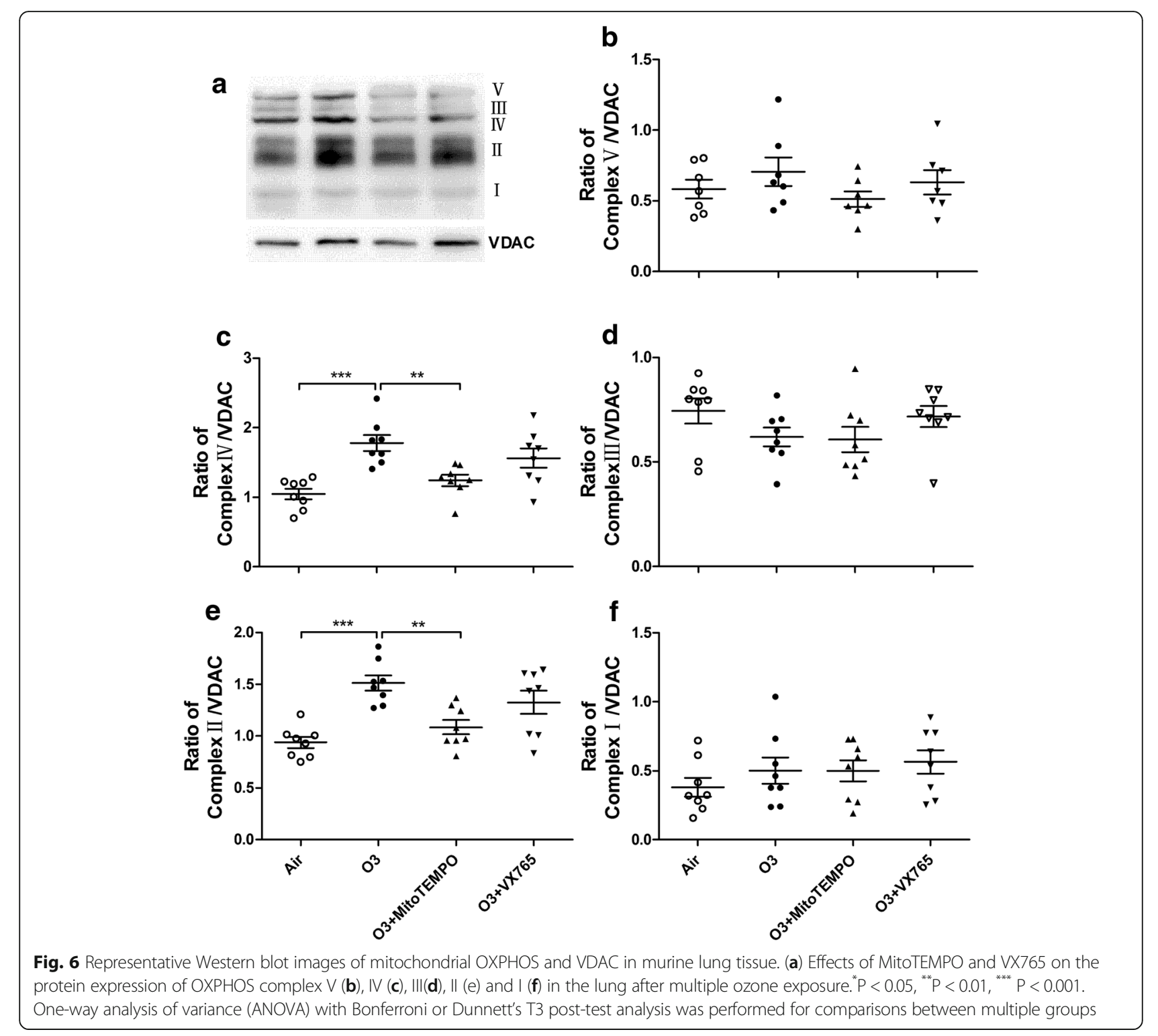

1. The data suggests that mtROS may not activate NLRP3 inflammasome-mediated ozone-induced emphysema.

Consistent with previous studies $[19,20]$, our experiments confirmed that multiple ozone exposure caused lung inflammation. This was reflected by increased numbers of total cells, macrophages, lymphocytes, neutrophils and eosinophils, and increased levels of $\mathrm{KC}, \mathrm{IL}-6$ and $\mathrm{IL}-1 \beta$ in BAL fluid, along with increased inflammatory scores in lung sections after ozone exposure. In our experiments, the dose of MitoTEMPO and VX765 was chosen according to previous studies [23, 24] and our own pilot data. MitoTEMPO failed to inhibit the recruitment of inflammatory cells following ozone exposure, but reduced $\mathrm{KC}$ and IL-6 levels in BAL fluid and inflammation scores in lung tissue. In contrast, VX765 reduced ozone effects on inflammatory cells, KC, IL- 6 and IL- $1 \beta$ in BAL fluid and inflammation scores in lung tissue. These findings indicate that both mtROS and NLRP3 played an important role in ozone-induced lung inflammation.

Ozone is a strong oxidizing agent, and can generate ROS and oxidative stress, which is a major mechanism in ozone-induced lung injury. Consistent with our previous study [20], the present study showed that multiple ozone exposure induced an increase in serum 8-OHdG, but not in BAL MDA. A recent study showed that increased mtROS was observed in lung macrophages after $72 \mathrm{~h}$ of $0.7 \mathrm{ppm}$ ozone exposure [25]. We here demonstrated that elevated mtROS levels in lung tissue of mouse by multiple ozone exposure. MitoTEMPO treatment reduced mtROS in cigarette smoke extract 

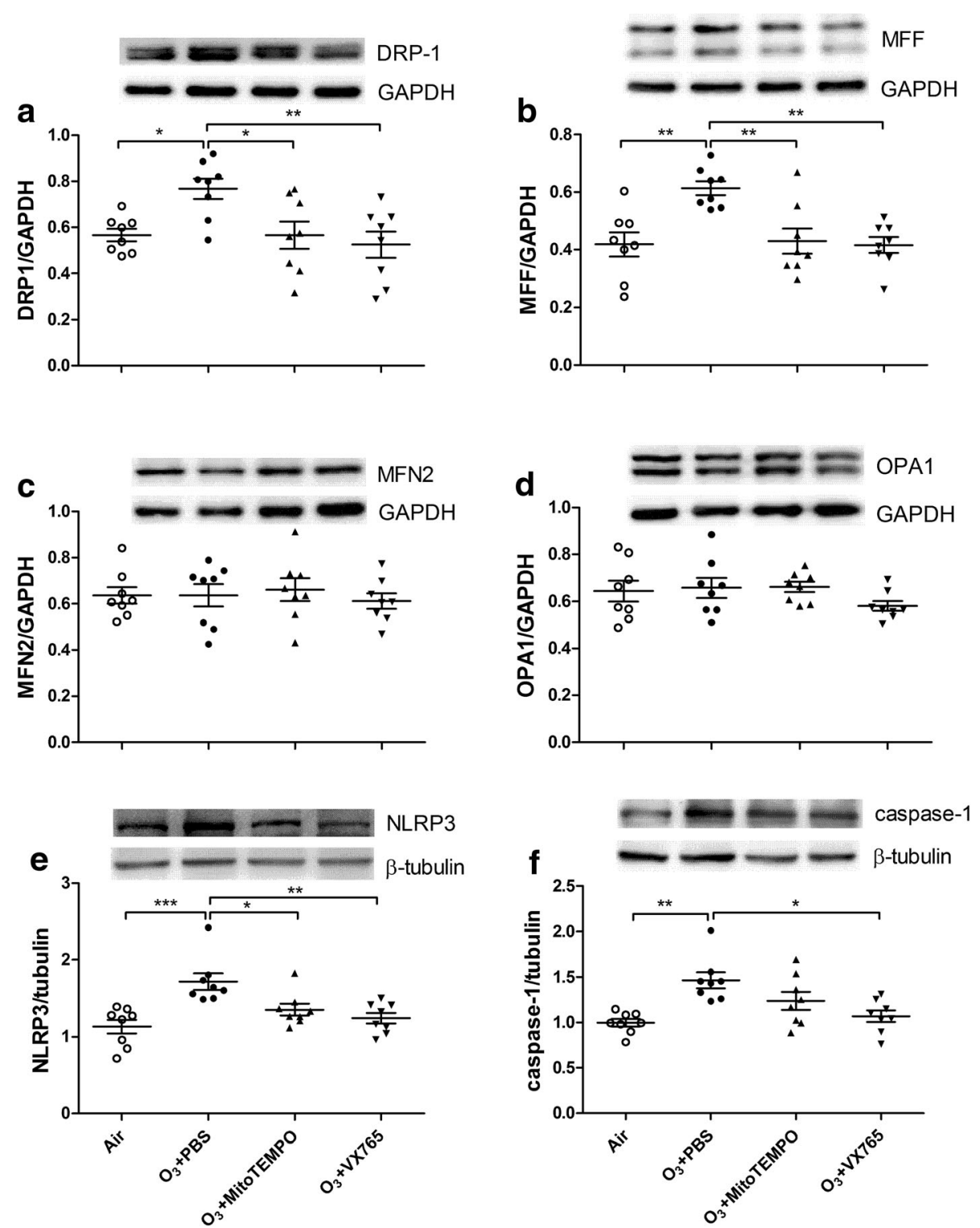

Fig. 7 Effects of MitoTEMPO and VX765 on the protein expression of mitochondrial fission/fusion-related proteins including DRP1 (a), MFF (b), MFN2 (c) and OPA1 (d), and NLRP3 (e) and caspse-1 (f) in the lung after multiple ozone exposure. Each panel shows representative Western blot. ${ }^{*} \mathrm{P}<0.05,{ }^{* *} \mathrm{P}<0.01,{ }^{* * *} \mathrm{P}<0.001$. One-way analysis of variance (ANOVA) with Bonferroni or Dunnett's $\mathrm{T3}$ post-test analysis was performed for comparisons between multiple groups

(CSE)-treated human lung fibroblast cells [26]. Our results showed that MitoTEMPO reduced 8-OHdG in serum and mtROS in lung tissue. Mitochondrial dysfunction including mtROS has been proposed to stimulate NLRP3 inflammasome activation [27] and activated NLRP3 inflammasome in turn may lead to mitochondrial damage [28]. In the present study, we observed that VX765 reduced serum 8-OHdG and lung mtROS, which could occur through inhibition of oxidative stress as a result of suppressing the activation of NLRP3 inflammasome.
Multiple ozone exposure-induced emphysema was confirmed by the increased mean linear intercept (Lm) in the lung sections, which indicates an increase in alveolar size. Further analysis showed that airway smooth muscle layer and total airway wall were increased in the ozone-exposed mice, compatible with the airway remodeling seen in patients with COPD [29]. In line with these, there were increases in the lung volume parameters including IC, FRC and TLC and in lung compliance (Cchord), and decreases in the airflow as indicated by decreases in the ratios of $\mathrm{FEV}_{25} / \mathrm{FVC}$ and $\mathrm{FEV}_{50} / \mathrm{FVC}$ in ozone-exposed 
Table 1 The effects of MitoTEMPO and VX-765 on ozone-induced lung inflammation and emphysema

\begin{tabular}{|c|c|c|}
\hline Parameter & MitoTEMPO & VX-765 \\
\hline IC, FRC, TLC, Cchord & $\mathrm{NE}$ & $\downarrow$ \\
\hline $\mathrm{FEV}_{25} / \mathrm{FVC}, \mathrm{FEV}_{50} / \mathrm{FVC}$ & NE & $\uparrow$ \\
\hline $\begin{array}{l}\text { Bronchoalveolar } \\
\text { lavage cells }\end{array}$ & NE & $\begin{array}{l}\downarrow \text { total cells,macrophages, } \\
\text { neutrophils,eosinophils }\end{array}$ \\
\hline Lung inflammation score & $\downarrow$ & $\downarrow$ \\
\hline Inflammatory cytokines & $\downarrow K C, I L-6$ & $\downarrow \| L-1 \beta, K C, I L-6$ \\
\hline Oxidative stress & $\begin{array}{l}\downarrow 8-\mathrm{OHdG} \text {, lung } \\
\text { mtROS }\end{array}$ & $\downarrow$ 8-OHdG,lung mtROS \\
\hline Mean linear intercept & $\mathrm{NE}$ & $\downarrow$ \\
\hline $\begin{array}{l}\text { Airway structure } \\
\text { components }\end{array}$ & $\mathrm{NE}$ & $\downarrow$ Wam/Pbm, Wat/Pbm \\
\hline Mitochondrial OXPHOS & $\downarrow$ complex II,IV & $\mathrm{NE}$ \\
\hline $\begin{array}{l}\text { Mitochondrial fission } \\
\text { protein }\end{array}$ & $\downarrow D R P 1, M F F$ & $\downarrow D R P 1, M F F$ \\
\hline $\begin{array}{l}\text { Mitochondrial fusion } \\
\text { protein }\end{array}$ & $\mathrm{NE}$ & $\mathrm{NE}$ \\
\hline NLRP3 protein & $\downarrow$ & $\downarrow$ \\
\hline Caspase-1 protein & $\mathrm{NE}$ & $\downarrow$ \\
\hline Caspase-1 activity & NE & $\downarrow$ \\
\hline
\end{tabular}

The effects of MitoTEMPO and VX-765 on lung function,lung inflammation (bronchoalveolar lavage cells, inflammation score,Inflammatory cytokines), oxidative stress, lung histology(mean linear intercept, airway structure components), mitochondrial changes (OXPHOS, fission- and fusion-related proteins) and expression and activity of NLRP3/caspase-1

$N E$ no effect $\downarrow$ decrease $\uparrow$ increase

mice. We observed that treatment with VX765, but not MitoTEMPO, prevented the development of emphysema, airway remodeling and airflow limitation in ozone-exposed mice. We interpreted these results as indicating that NLPR3 activation is through other mechanisms apart from mtROS as far as the development of these ozone-induced features is concerned.

Mitochondrial dysfunction including increased mtROS production and impaired OXPHOS has been linked to the pathogenesis of COPD [30]. The expression of mitochondrial OXPHOS complexes was increased in BEAS-2B cells by long term CSE exposure as well as in bronchial epithelial cells from COPD patients [31]. The increased expression of mitochondrial complex II and IV in ozone-exposed mice may indicate increased capacity of OXPHOS to produce mtROS, as the treatment with MitoTEMPO inhibited the expression of both complexes as well as the levels of mtROS.

Mitochondria are regulated through cycles of fusion and fission. Mitochondrial fussion is induced by acute oxidative stress, which possibly acts as defense against ROS, while mitochondrial fission is induced by more prolonged oxidative stress and mitochondrial dysfunction, which facilitates removal of damaged mitochondria and induction of apoptosis [7]. Increased expression of DRP1 was found in a mouse emphysema model induced by over-expression of cathepsin $\mathrm{E}$ where inhibition of DRP1 prevented cell apoptosis and emphysema [32]. Similarly, in the present study, mitochondrial fusion-related proteins were unchanged whilst mitochondrial fission-related proteins were enhanced. This indicates that increased mitochondrial fission occurs in the lung tissue of ozone-induced lung inflammation and emphysema.

The activity of NLRP3 was elevated in various lung inflammation models. For example, IL-1 $\beta$, IL- 6 and TNF- $\alpha$ were elevated in BAL fluid and NLRP3 was activated in lung tissue of lipopolysaccharide(LPS)-induced lung injury model [33]. IL-1 $\beta$ concentrations in BAL fluid and NLRP3 mRNA in lung tissue were increased in $\mathrm{PM}_{2.5}$-induced acute lung inflammation model [34]. Most animal models have shown a crucial role for the NLRP3 inflammasome in the inflammatory and immune responses in COPD. However, the data in humans have been more variable [35]. A recent study using an anti-IL-1R mAb (MED18968) was ineffective in COPD [36]. More long-term clinical trials with canakinumab or NLRP3-specific agents are needed [37]. The mtROS inhibitor NecroX-5 reduces NLRP3 inflammasome activation in an allergic mouse model [38]. Our study showed that MitoTEMPO inhibited the expression of NLRP3 in lung tissue, which may indicate that mtROS is the upstream of NLRP3 and that inhibition of mtROS can lead to a reduction of NLRP3 expression. The present study also showed that VX765 inhibited the expression of both NLRP3 and caspase-1 in lung tissue.

\section{Conclusions}

In summary, these results from our study support our hypothesis that mtROS and NLRP3 inflammasome are involved in ozone-induced lung inflammation, while only NLRP3 inflammasome is involved in ozone-induced lung emphysema. This suggests that the latter is independent of mtROS and may be activated by non-mtROS mechanisms.

\section{Additional file}

Additional file 1: The effect of 3-week treatment with MitoTEMPO or VX765 on air-exposed mice. (DOC 5569 kb)

\section{Abbreviations}

8-OHdG: 8-hydroxy-2'-deoxyguanosine; ATP: Adenosine triphosphate; BAL: Bronchoalveolar lavage; Cchord: Chord compliance; COPD: Chronic obstructive pulmonary disease; CSE: Cigarette smoke extract;

CXCL1: Chemokine (C-X-C motif) ligand 1; DRP1: Dynamin related protein; $\mathrm{FEV}_{25}$ : Forced expiratory volume at 25 milliseconds; FEV $_{50}$ : Forced expiratory volume at 50 milliseconds; FRC: Functional residual capacity; FVC: Forced

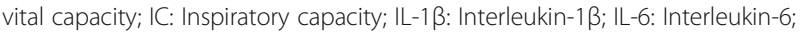
LPS: Lipopolysaccharide; MDA: Malondialdehyde; MFF: Mitochondrial fission factor; mtROS: Mitochondrial ROS; NE: No effect; NLR: Nucleotide binding domain leucine-rich repeat-containing receptor; OPA1: Optic atrophy protein 
1; PBS: Phosphate buffered saline; ROS: Reactive oxygen species; TLC: Total lung capacity

\section{Acknowledgements}

Not applicable.

\section{Funding}

This work was supported by the Key International (Regional) Cooperative Project of National Natural Science Foundation of China (No.51420105010), the National Nature Science Foundation of China (Project No.81470218) and the Shanghai Pujiang Program (No.14PJD027).

\section{Availability of data and materials}

The data that support the findings of this study are available from the corresponding author upon reasonable request.

\section{Authors' contributions}

FL, IMA, KFC, XZ conceived and designed the study. FL, MX, MW, HW, LW carried out the animal exposure and measured lung function. FL, MX, MW, $H Z, Y C$ performed pathological analysis and molecular biological experiments. JG, JZ performed HPLC analysis. FL, MX, MW prepared the manuscript. FL, IMA KFC, XZ analyzed data and contributed to manuscript revision. All authors read and approved the final manuscript.

\section{Ethics approval and consent to participate}

The experimental procedures were approved by the Laboratory Animal Ethics Committee of Shanghai Chest Hospital.

\section{Consent for publication}

Not applicable.

\section{Competing interests}

The authors declare that they have no competing interests.

\section{Publisher's Note}

Springer Nature remains neutral with regard to jurisdictional claims in published maps and institutional affiliations.

\section{Author details \\ 'Department of Pulmonary Medicine, Shanghai Chest Hospital, Shanghai Jiaotong University, Shanghai 200030, People's Republic of China. 2Department of Respiratory Medicine, Shanghai First People's Hospital, Shanghai Jiaotong University, No.100, Haining Road, Shanghai 200080, China. ${ }^{3}$ Duke Global Health Institute and Nicholas School of the Environment, Duke University, Durham, NC 27708, USA. ${ }^{4}$ College of Environmental Sciences and Engineering and BIC-ESAT, Peking University, Beijing 100871, People's Republic of China. ${ }^{5} \mathrm{Global}$ Health Research Center, Duke Kunshan University, Kunshan, Jiangsu 215316, People's Republic of China. ${ }^{6}$ Airway Disease Section, National Heart and Lung Institute, Imperial College London, London SW3 6LY, UK. ${ }^{7}$ Priority Research Centre for Asthma and Respiratory Disease, Hunter Medical Research Institute, University of Newcastle, Newcastle, NSW 2305, Australia.}

\section{Received: 23 August 2018 Accepted: 7 November 2018}

\section{Published online: 22 November 2018}

\section{References}

1. Adeloye D, Chua S, Lee C, Basquill C, Papana A, Theodoratou E, Nair H, Gasevic D, Sridhar D, Campbell H, Chan KY, Sheikh A, Rudan I. Global Health epidemiology reference group (GHERG). Global and regional estimates of COPD prevalence: systematic review and meta-analysis. J Glob Health. 2015;5(2):020415.

2. Vogelmeier CF, Criner GJ, Martinez FJ, Anzueto A, Barnes PJ, Bourbeau J, Celli BR, Chen R, Decramer M, Fabbri LM, Frith P, Halpin DM, López Varela MV, Nishimura M, Roche N, Rodriguez-Roisin R, Sin DD, Singh D, Stockley R, Vestbo J, Wedzicha JA, Agustí A. Global strategy for the diagnosis, management, and prevention of chronic obstructive lung disease 2017 report. GOLD executive summary. Am J Respir Crit Care Med. 2017;195(5):557-82.

3. Kirkham PA, Barnes PJ. Oxidative stress in COPD. Chest. 2013;144(1):266-73.

4. Archer SL. Mitochondrial dynamics- mitochondrial fission and fusion in human diseases. N Engl J Med. 2013;369(23):2236-51.
5. Białas AJ, Sitarek P, Miłkowska-Dymanowska J, Piotrowski WJ, Górski P. The role of mitochondria and oxidative/Antioxidative imbalance in pathobiology of chronic obstructive pulmonary disease. Oxidative Med Cell Longev. 2016;2016:7808576.

6. Yue L, Yao H. Mitochondrial dysfunction in inflammatory responses and cellular senescence: pathogenesis and pharmacological targets for chronic lung diseases. Br J Pharmacol. 2016;173(15):2305-18.

7. Michaeloudes C, Bhavsar PK, Mumby S, Chung KF, Adcock IM. Dealing with stress: defective metabolic adaptation in chronic obstructive pulmonary disease pathogenesis. Ann Am Thorac Sco. 2017;14(supplement 5):S374-82.

8. Prakash YS, Pabelick CM, Sieck GC. Mitochondrial dysfunction in airway disease. Chest. 2017;152(3):618-26.

9. Lee S, Suh GY, Ryter SW, Choi AM. Regulation and function of the nucleotide binding domain leucine-rich repeat-containing receptor, pyrin domain-Containing-3 Inflammasome in lung disease. Am J Respir Cell Mol Biol. 2016;54(2):151-60.

10. Jo EK, Kim JK, Shin DM, Sasakawa C. Molecular mechanisms regulating NLRP3 inflammasome activation. Cell Mol Immunol. 2016;13(2):148-59.

11. Zhou R, Yazdi AS, Menu P, Tschopp J. A role for mitochondria in NLRP3 inflammasome activation. Nature. 2011;469(7329):221-5.

12. Shimada K, Crother TR, Karlin J, Dagvadorj J, Chiba N, Chen S, Ramanujan VK, Wolf A, Vergnes L, Ojcius DM, Rentsendorj A, Vargas M, Guerrero C, Wang Y, Fitzgerald KA, Underhill DM, Town T, Arditi M. Oxidized mitochondrial DNA activates the NLRP3 inflammasome during apoptosis. Immunity. 2012;36(3):401-14.

13. Mariathasan S, Weiss DS, Newton K, McBride J, O'Rourke K, Roose-Girma M, Lee WP, Weinrauch Y, Monack DM, Dixit VM. Cryopyrin activates the inflammasome in response to toxins and ATP. Nature. 2006:440(7081):228-32.

14. Yamasaki K, Muto J, Taylor KR, Cogen AL, Audish D, Bertin J, Grant EP, Coyle AJ, Misaghi A, Hoffman HM, Gallo RL. NLRP3/cryopyrin is necessary for interleukin1 beta (IL-1 beta) release in response to hyaluronan, an endogenous trigger of inflammation in response to injury. J Biol Chem. 2009;284(19):12762-71.

15. Li H, Ambade A, Re F. Cutting edge: necrosis activates the NLRP3 inflammasome. J Immunol. 2009;183(3):1528-32.

16. Kousha T, Rowe BH. Ambient ozone and emergency department visits due to lower respiratory condition. Int J Occup Med Environ Health. 2014;27(1):50-9.

17. Ghanbari Ghozikali M, Heibati B, Naddafi K, Kloog I, Oliveri Conti G, Polosa R, Ferrante M. Evaluation of chronic obstructive pulmonary disease (COPD) attributed to atmospheric O3, NO2, and SO2 using air Q model (2011-2012 year). Environ Res. 2016;144(Pt A):99-105.

18. Ji M, Cohan DS, Bell ML. Meta-analysis of the association between short-term exposure to ambient ozone and respiratory hospital admissions. Environ Res Lett. 2011;6(2).

19. Li F, Wiegman C, Seiffert JM, Zhu J, Clarke C, Chang Y, Bhavsar P, Adcock I, Zhang J, Zhou X, Chung KF. Effects of N-acetylcysteine in ozone-induced chronic obstructive pulmonary disease model. PLoS One. 2013;8(11):e80782.

20. Li F, Zhang P, Zhang M, Liang L, Sun X, Li M, Tang Y, Bao A, Gong J, Zhang J, Adcock I, Chung KF, Zhou X. Hydrogen sulfide prevents and partially reverses ozone-induced features of lung inflammation and emphysema in mice. Am J Respir Cell Mol Biol. 2016;55(1):72-81.

21. Chen O, Ye Z, Cao Z, Manaenko A, Ning K, Zhai X, Zhang R, Zhang T, Chen $X$, Liu W, Sun X. Methane attenuates myocardial ischemia injury in rats through anti-oxidative, anti-apoptoic and anti-inflammatory actions. Free Radic Biol Med. 2016;90:1-11.

22. Jiang $L$, Zhang $L$, Kang K, Fei D, Gong R, Cao Y, Pan S, Zhao M, Zhao $M$. Resveratrol ameliorates LPS-induced acute lung injury via NLRP3 inflammasome modulation. Biomed Pharmacother. 2016;84:130-8.

23. Patil NK, Parajuli N, MacMillan-Crow LA, Mayeux PR. Inactivation of renal mitochondrial respiratory complexes and manganese superoxide dismutase during sepsis: mitochondria-targeted antioxidant mitigates injury. Am J Physiol Renal Physiol. 2014;306(7):F734-43.

24. Bassil F, Fernagut PO, Bezard E, Pruvost A, Leste-Lasserre T, Hoang QQ, Ringe D, Petsko GA, Meissner WG. Reducing C-terminal truncation mitigates synucleinopathy and neurodegeneration in a transgenic model of multiple system atrophy. Proc Natl Acad Sci U S A. 2016;113(34):9593-8.

25. Che L, Jin Y, Zhang C, Lai T, Zhou H, Xia L, Tian B, Zhao Y, Liu J, Wu Y, Wu Y, Du J, Li W, Ying S, Chen Z, Shen H. Ozone-induced IL-17A and neutrophilic airway inflammation is orchestrated by the caspase-1-IL-1 cascade. Sci Rep. 2016;6:18680.

26. Ahmad T, Sundar IK, Lerner CA, Gerloff J, Tormos AM, Yao H, Rahman I. Impaired mitophagy leads to cigarette smoke stress-induced cellular senescence: implications for chronic obstructive pulmonary disease. FASEB J. 2015;29(7):2912-29. 
27. Gurung P, Lukens JR, Kanneganti TD. Mitochondria: diversity in the regulation of the NLRP3 inflammasome. Trends Mol Med. 2015;21 (3):193-201.

28. Yu J, Nagasu H, Murakami T, Hoang H, Broderick L, Hoffman HM, Horng T. Inflammasome activation leads to Caspase-1-dependent mitochondrial damage and block of mitophagy. Proc Natl Acad Sci U S A. 2014; 111(43):15514-9.

29. Sohal SS, Ward C, Danial W, Wood-Baker R, Walters EH. Recent advances in understanding inflammation and remodeling in the airways in chronic obstructive pulmonary disease. Expert Rev Respir Med. 2013;7(3):275-88.

30. Hara H, Araya J, Ito S, Kobayashi K, Takasaka N, Yoshii Y, Wakui H, Kojima J, Shimizu K, Numata T, Kawaishi M, Kamiya N, Odaka M, Morikawa T, Kaneko Y, Nakayama K, Kuwano K. Mitochondrial fragmentation in cigarette smoke-induced bronchial epithelial cell senescence. Am J Physiol Lung Cell Mol Physiol. 2013; 305(10):L737-46.

31. Hoffmann RF, Zarrintan S, Brandenburg SM, Kol A, de Bruin HG, Jafari S, Dijk F, Kalicharan D, Kelders M, Gosker HR, Ten Hacken NH, van der Want JJ, van Oosterhout AJ, Heijink IH. Prolonged cigarette smoke exposure alters mitochondrial structure and function in airway epithelial cells. Respir Res. 2013;14:97.

32. Zhang X, Shan P, Homer R, Homer R, Zhang Y, Petrache I, Mannam P, Lee PJ. Cathepsin E promotes pulmonary emphysema via mitochondrial fission. Am J Pathol. 2014;184(10):2730-41.

33. Zhang A, Pan W, LV J, Wu H. Protective effect of amygdalin on LPS-induced acute lung injury by inhibiting NF-KB and NLRP3 signaling pathways. Inflammation. 2017: 40(3):745-51.

34. Wang $H$, Song $L$, Ju W, Wang $X$, Dong L, Zhang Y, Ya P, Yang C, Li F. The acute airway inflammation induced by PM2.5 exposure and the treatment of essential oils in Balb/c mice. Sci Rep. 2017;7:44256.

35. Li C, Zhihong H, Wenlong L, Xiaoyan L, Qing C, Wenzhi L, Siming X, Shengming $L$. The nucleotide-binding oligomerization domain-like receptor family pyrin domain-containing 3 Inflammasome regulates bronchial epithelial cell injury and Proapoptosis after exposure to biomass fuel smoke. Am J Respir Cell Mol Biol. 2016;55(6):815-24.

36. Calverley PMA, Sethi S, Dawson M, Ward CK, Finch DK, Penney M, Newbold P, Van der Merwe R. A randomised, placebo-controlled trial of anti-interleukin-1 receptor monoclonal antibody MEDI8968 in chronic obstructive pulmonary disease. Respir Res. 2017;18(1):153.

37. Rogliani P, Calzetta L, Ora J, Matera MG. Canakinumab for the treatment of chronic obstructive pulmonary disease. Pulm Pharmacol Ther. 2015;31:15-27.

38. Kim SR, Kim DI, Kim SH, Lee H, Lee KS, Cho SH, Lee YC. NLRP3 inflammasome activation by mitochondrial ROS in bronchial epithelial cells is required for allergic inflammation. Cell Death Dis. 2014;5:e1498.

Ready to submit your research? Choose BMC and benefit from:

- fast, convenient online submission

- thorough peer review by experienced researchers in your field

- rapid publication on acceptance

- support for research data, including large and complex data types

- gold Open Access which fosters wider collaboration and increased citations

- maximum visibility for your research: over $100 \mathrm{M}$ website views per year

At BMC, research is always in progress.

Learn more biomedcentral.com/submissions 